\title{
Research on Scenic Distributing Flow Scheduling and Security Application Based on Matlab
}

\author{
Wenling Liu ${ }^{1}$, Saifeng Zhuo ${ }^{1}$, Kun Zhang ${ }^{2}$, and Weixin Wang ${ }^{2}$ \\ ${ }^{1}$ School of Information Science and Technology, Taishan University (TSU), Shandong 271021, China \\ ${ }^{2}$ School of mathematics and statistics, Taishan University (TSU), Shandong 271021, China
}

Keywords: Matlab, shunt scheduling, loading rate.

\begin{abstract}
This paper uses Matlab and a series of mathematical modeling knowledge to start research. According to the scenic spot load rate situation, various diversion resources should be reasonably allocated to ensure that attractions with high loading rates have preferential access to diversion resources. It is more effective to use the relationship between attractions and resource points to rationalize the dispatch, rather than one-sided scheduling of resources, number of people, distance, and diversion capability. In the tourist peak areas, there are some phenomena such as high traffic volume, high crowding intensity and high load rate of popular spots. The demand for tourist diversion of scenic spots refers to tourists using transport or walking to go to the next tourist attraction, which has a part of the demand for transport and pedestrian roads. Based on the analysis of this distributive resource capability, the gravity-distribution dispatching model of diversion resources for tourists' traveling movement will be analyzed in the space-time dimension.
\end{abstract}

\section{Research Background}

During the May 1st holiday, Taishan once again welcomed the passenger flow peak. Before the arrival of the small holiday, the Taishan Scenic Area Information Center integrated the Wuli passenger flow data of the calendar year, the number of people entering the mountain on March 15th, the online sales data of the scenic area network, the data of Ctrip, Baidu maps, the reservation of the scenic spot team, and other aspects. , Made reports on the analysis of the passenger flow during the "May 1st" holiday and "March 15" folk festival. The situation of full-blown tourists in Taishan scenic spot appears before and after sunrise in the morning. Problems to be solved:

(1) Collect relevant data to analyze the passenger flow of Mount Tai Scenic Area; (2) Formulate a detailed diversion plan using mathematical modeling, and provide detailed measures to be thorough; (3) Solve downhill down The "head-on collision" problem of tourists; (4) Combining the diversion plan, formulate a detailed security plan, and use the data to explain the scientific nature of the proposed method.

Red Gate on foot Dengtian Road, Tiandi Plaza by car Dengtian Road, Taohuayuan by car Dengtian Road, Tiandeng Peak on foot Deng Tian Road: Taohua Mountain hiking route (the most convenient); Red Gate Road hiking route (most classic); Tianwai Village car mountaineering route (the most troublesome); day candle peak climbing line (the most primitive natural); Taishan East Road (Hongmen Road) summit route;

Taishan West Road (Tiandi Square) is the top route and is available for route selection: Temple $\rightarrow$ Red Gate $\rightarrow$ Zhongtian Gate $\rightarrow$ 18th Disk $\rightarrow$ Nantianmen $\rightarrow$ Jade Emperor Roof; Tiandi Square $\rightarrow$ Zhongtian Gate $\rightarrow$ Nantianmen; Picking Flowers $\rightarrow$ Taohuayuan $\rightarrow$ Nantianmen; Tianzhufeng $\rightarrow$ Yuhuangding; $\quad$ Mingtang $\quad$ Park $\rightarrow$ Tianlong $\quad$ Reservoir $\rightarrow$ Patio $\quad$ Bay $\rightarrow$ Sales Shelf $\rightarrow$ Yuhuangding.

\section{Problem Analysis and Explanation}

For the first problem, we can contrast the examples, according to the Taishan Scenic Area tourist 
visits over the years, as well as Taishan tickets sold, analysis and compariso.

For the second problem, according to the specific data collected, establish a mathematical model, and then test and analyze the model, and predict the situation of the flow of people in the scenic spot, and formulate appropriate diversion strategies to avoid the "head-on collision" of tourists going down the mountain. The phenomenon. And develop a series of corresponding emergency measures.

The question is raised: Assume that the Taishan scenery area is in good condition on the day and no natural disasters occur; assume that the tourists are traveling at the same rate; assume that the tourists are going down the mountain at the same speed; assume that the speed of the shuttle bus picking up the tourists is the same and the passenger capacity is the same. The relevant study symbol description is shown in the following table:

Tab 1 Research Symbols

\begin{tabular}{cc}
\hline t & Stay time \\
\hline A & Attractions collection \\
N & Number of visitors \\
$\mathrm{C}$ & Natural capacity \\
$\mathrm{B}$ & Tourists collection \\
$\mathrm{V}$ & Speed \\
$\mathrm{Dm}$ & Instantaneous static capacity \\
$\mathrm{L}$ & Pedestrian path length \\
$\mathrm{Vj}$ & Vehicle speed \\
$\mathrm{Ri}$ & Distance from vehicle to diverging attraction \\
$\mathrm{Cj}$ & Remaining passenger capacity \\
$\mathrm{D}$ & A reasonable distance between pedestrians \\
$\mathrm{W}$ & Pavement width \\
$\mathrm{Vi}$ & Average travel speed of visitors \\
\hline
\end{tabular}

\section{Establishment of Model Indicators}

\subsection{Analysis of passenger flow in the area and its demand.}

Assume the space-time load rate of scenic spot $j$ at s,then $R_{j}^{?}$ It looks like this:

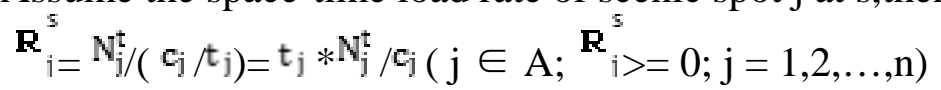

Where $A$ is a collection of attractions and, ${ }^{c}$. For the natural capacity of the site $j$; ${ }^{t_{j}}$ Average length of stay for travelers in scenic spots $\mathrm{j}, \mathrm{N}_{\mathrm{j}}^{1}$ Number of visitors to attraction $\mathrm{j}$ at $\mathrm{t}$.

$$
\mathrm{N}_{\mathrm{j}}^{1}=\sum_{\mathrm{i} \in \mathrm{B}} \mathrm{x}_{\mathrm{ij}}
$$

when $\mathrm{t}-\mathrm{s}_{\mathrm{ij}} \leq 1, \mathrm{x}_{\mathrm{ij}}=1$,or $=0$ ), B Set for tourists.

\subsection{Vehicle Distributive Capacity}

Since the types of vehicles used for tourist diversion in scenic spots are generally fixed, the carrying capacity is generally uniform, and the driving speed of the vehicles to divert target spots can be set as the average value. The ability to divert traffic from a vehicle is actually determined by the remaining capacity and speed of the vehicle. Under the assumption that the initial state of the vehicle is stationary, the diversion capability of the vehicle $\mathrm{j}$ at time $\mathrm{t}, \mathrm{K}_{\mathrm{j}}=\mathrm{C}_{\mathrm{i}}\left(\mathrm{V}_{\mathrm{l}} / \mathrm{V}_{\mathrm{i}}\right.$ ) among them $\mathrm{C}_{\mathrm{i}}$ For the remaining passenger capacity of the vehicle $\mathrm{j}$ at $\mathrm{t}, \mathrm{vj}$ is the speed of the vehicle, and $\mathrm{rj}$ is the distance from the vehicle to the diversion demand attraction.when $\mathrm{V}_{\mathrm{i}}$ A value of 0 indicates that the vehicle is at a desired point of interest.

The mathematical model of vehicle speed and traffic density is established below:

$\mathrm{v}=\mathrm{Vm} *(1-\mathrm{k} / \mathrm{Km})$, 
$\mathrm{q}=\mathrm{v}^{*} \mathrm{k}$,

$\mathrm{v}=\mathrm{Vm} * \ln (\mathrm{Km} / \mathrm{k})$,

It can be seen that when the density $\mathrm{k}=0$, the vehicle speed reaches the maximum, when the density $\mathrm{k}=\mathrm{Km}$, ie when the traffic density reaches the maximum, $\mathrm{v}=0$. The above mathematical model is applicable to the situation of moderate traffic density. In order to study the situation with large traffic density, the model correction is shown below. In order to more clearly analyze the relationship between vehicle speed and traffic density, use matlab software to draw.

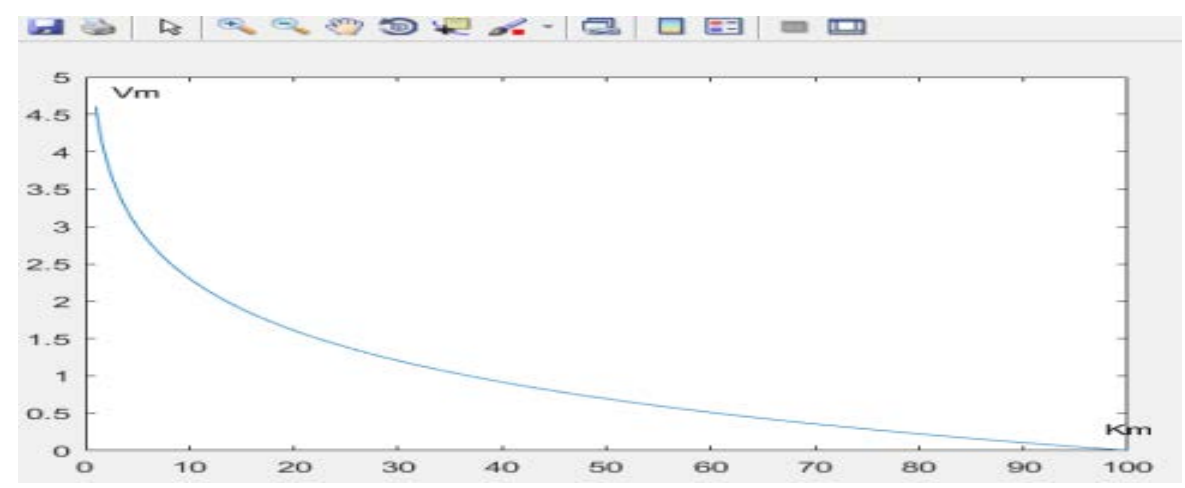

Fig. 1 Matlab drawing

\subsection{Tourist attraction gravitational flow dispatching model}

In the following, we will analyze and study the gravitational shunting and dispatching model of diversion resources for tourists' traveling movement in the space-time dimension. This also provides appropriate decision support for real-time scheduling of tourist distribution. The gravity-distribution gravity model of tourists in scenic spots is shown below. The gravitational measure value between the point $i$ that is to be diverted at time $t$ and the resource point $j$ that is diverted $\left.{ }^{F}{ }_{i=G}^{t}=G_{p}^{t}\right)^{\alpha 1}\left(K_{p}^{t}\right)^{\alpha 2}$ $/ \mathrm{Z}_{\mathrm{ij}}^{\alpha 3}, \mathrm{R}_{\mathrm{i}}^{1}, \mathrm{~K}_{\mathrm{j}}^{4}$ Degree constant value, $\mathrm{Z}_{\mathrm{ij}}$ For the diversion of the attractions i to the diversion of resource points $j$, the scheduling resistance. The dispatching resistance reflects the resource balance between the diverted resources and the demand spots. Factors that affect this resistance are usually the diversion of the line between attractions, road conditions, and diversion costs. For the sake of smooth research, we can convert the scheduling resistance into the time cost of component flow scheduling, and use cost, time, and other comprehensive generalized costs as parameters. Dispatch resistance function $Z_{\mathrm{i}}=a_{1} * t_{i j}+a_{2} * E_{i j}+a_{3} * \delta$,among them ${ }^{t_{i j}}$ It is the time when the split resource node $j$ reaches the demand point of the diversion $\mathrm{i}, \mathrm{E}_{\mathrm{i}}$ It is the cost of completing the scheduling task for the scenic spot $i$ that reaches the diversion demand from the diverging resource node $j, \delta$ is the penalty for diversion, such as convenience, viewing degree, comfort, safety performance, etc. a1,a2,a3 It is the pre-coefficient of resistance. Gravity diversion scheduling algorithm design for tourists at the six peaks.

\section{Model Establishment and Solution}

\subsection{Mathematical model of the algorithm}

If the weighting of the cost of the diversion and the satisfaction of the tourists is regarded as equally important, then the objective function is min

$$
\sum_{i} i \in A \Gamma_{i} i \in s\left(\mathrm{R}_{\mathrm{i} \cdot \times}^{\mathrm{t}} C_{i_{i}}+\varepsilon \mathrm{M}\left(t_{i_{i}}\right)\right)
$$

Where $\mathrm{A}$ is the set of demand points, $\mathrm{S}$ is the set of divergent resource points, and Rt ij is the distance between resource point $i$ at $t$ and the required sight point $j$. In this paper, for the purpose of facilitating the study, we take the Euclidean distance $R t i j=\left\|X_{i}(t), X_{i}(t)\right\|, c_{i j}$ The cost of diversion for the distance from the resource node $i$ to the unit of the demand attraction $\mathrm{j}$ is mainly represented by 
the transportation costs of various vehicles of the landscape and the maintenance costs of the pedestrian road.ePenalty coefficient, $\mathrm{M}\left(t_{i_{\star}}\right)$ Distribute schedules for visitors at waiting time .Satisfaction $t_{i_{\alpha}}$

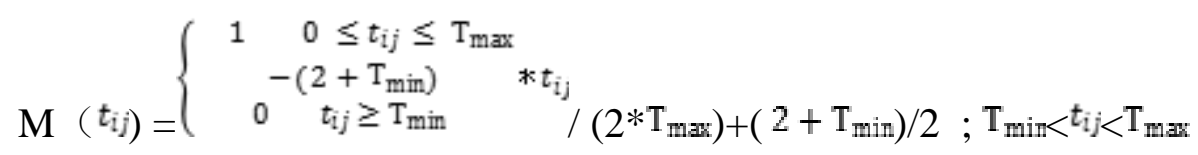

In equation (4), Tmax is the longest waiting time for satisfaction degree 1 , and Tmin is the shortest waiting time for satisfaction degree zero. Psychologists usually refer to the comprehensive reflection of time in people's psychology as time perception or time experience, and the guests also experience time perception or time experience in the process of experience. But at the same time, there is also time satisfaction.

\subsection{Algorithmic description}

The algorithm for the issue of diversion scheduling for tourists can be mainly divided into the following steps. Predict the load rate of tourist attractions at the next time $t$, and generate corresponding demand spots, According to the size of space-time load rates of tourist attractions, Calculate the value of each site's attractions and all the diversion resource nodes' gravitational measure, The corresponding diversion resources are matched accordingly, Distribute resources, update relevant tourist information, check whether the dispatching task can be completed, and output the corresponding dispatching plan.

The first step is to generate demand spots. Based on historical data of tourist flow distribution of tourist attractions and information on recent sights and visitor arrival time, departure time, waiting time, and other information, the distribution of tourists in future scenic spots is predicted, so that the space-time load rate of scenic spot attractions in future can be obtained, and then the attractions can be determined. Diversion demand conditions.

The second step is the gravitational measure calculation. The size of space-time load ratios of demand spots will be sorted in descending order. According to the principle of priority matching resources for tourist attractions with large load rates, gravitational models will be used to calculate the demand spots and known resource nodes, so as to obtain each resource node and Resource matching between demand spots.

The third step is to divert resources to re-test. In the process of allocating dispatch resources to a demand spot, there will be a case where the same gravity measurement value of the demand spot is different for different resource points. When this happens, calculate the value of the objective function of the demand spot and the different diversion resource points, and take the best one as the diversion scheduling scheme, and use the utility value of the objective function to further determine the optimal matching degree between the resource and the demand.

\subsection{Solution to Problem}

Through the disaster monitoring and management of the whole process, with the guidance of pre-warning and post-event emergency management and control, a crowd-prevention accident prevention system covering the entire process of Mount Tai's play is constructed. The management of the entire process of prevention of crowded accidents in Taishan scenic spot is based on a centralized information platform and a centralized command structure. Starting from the perspective of prevention, with the implementation of an effective supervision and control information system and a rapid response evacuation and rescue system, it will provide security for the smooth holding of Taishan scenic spot tourism. Looking forward to the future work and building an effective crowd protection accident prevention system for tourist tourists in Taishan, we need to strengthen the following aspects:

(1) The planned diversion plan for the population. In order to prevent the excessive concentration of large-scale social activities leading to the occurrence of various destructive outcomes, it is necessary to implement a series of methods for pricing and traffic control to decentralize the 
concentrated population and avoid the occurrence of large-scale crowding.

(2) Establish a real-time warning system for population distribution. Systematic and real-time grasping and analysis of the surrounding people flow, and early warning of the peak of people flow, guiding, diverting and evacuating people flow according to warning and warning information and related plans.

Taishan Scenic Area Command Center needs to do a good job of preventing the use of command vehicles and electronic access control, traffic statistics, unmanned aerial photography and other "digital Taishan" information platform, timely dynamics, advanced analysis and analysis to resolve short-term tourist congestion problems; fully activated 9 diversion lanes were coordinated, and at the same time, nearly 500 police officers and men were coordinated to quickly divert and maintain order; timely release of relevant information through electronic screens, radio, media, etc.

Taishan Mountain Scenic Area starts the I-level response mode during the holiday period, establishes a field command center in Zhongtian Gate, uses digital monitoring systems, drone cruise equipment and other advanced means to provide critical nodes, critical time passenger flow information, real-time statistics of HD video surveillance probes, and monitoring passenger flow Changes, timely and effective disposal of various types of emergencies. Before the holiday, we will renovate the diversion tunnel of "Wusong Pavilion-Fangtaizi”, "Hongdelou-Grand Platform-Sunview Peak", and completely solve the "head-on collision" problem of mountainous and mountainous tourists.

\section{Conclusions}

The real-time statistics of the passenger flow can provide the real-time passenger flow situation to the scenic spot managers. Since each scenic spot has its own carrying capacity, it is possible to adopt current-limiting measures in time at the peak. Through the statistics of passenger flow, such as time, day, week, month, and year, such as entrances and exits, areas, etc., the flow data of scenic spots and scenic spots are measured.

Advantages: The originality is very strong, and most of the models in the article are self-derivative. The calculation of the model adopts professional mathematics software with high reliability; the established model can be closely linked with the actual situation, and the problem is solved by combining the actual situation, so that the model has good versatility and popularization; the model involves Many quantitative factors were analyzed to make the paper more persuasive.

Disadvantages: The constraints of the planning model are a bit simple; there are too many variables introduced in the process of model building, which is not conducive to programming; there are too many calculation projects, there is a great deal of connection, prone to calculation errors, resulting in global errors; not all the influencing factors can be included in the calculation.

\section{Acknowledgments}

This research has been partially supported by Taishan university's young teachers' research fund project(No.: QN-01-201702), the national Spark Program project (No.:2014GA740055), science and technology development project of Tai'an city (No.: 20140630-6), science and technology plan project of Shandong province (No.: J14LN68). This research has been partially supported by Shandong province undergraduate colleges and universities Research project on teaching reform; Shandong province soft science research plan; Ministry of education cooperative education comprehensive reform project. We like to express our appreciation for the valuable suggestions from the referee and the editor of this journal which significantly improved the quality of the presented paper. 


\section{References}

[1] Hu Zhiying, Ye Minghai, Research on crowd protection accident prevention system for large-scale social activities, Tongji University, School of Economics and Management, Shanghai

[2] Sun Chao, Ren Yanbin, Management of crowds in large-scale social activities, Beijing

[3] Guo Wei. Inbound Tourism: An Empirical Study Based on Gravity Model [J]. Tourism Journal, 2007( 22) : 30-34.

[4] Liu Zhusheng. Study on Time-space Diversion Navigation Management Theory and Vehicle Dispatching of Tourists in Scenic Spots [D]. Chengdu: Sichuan University

[5] Feng Gang. Scenic visitor time and space diversion navigation management [M]. Beijing: Peking University Press, China Forestry Publishing House, 2011: 59-121. 